\title{
Understanding the Patient Perception of Statin Experience: A Qualitative Study
}

\author{
Michal Vrablik - Alberico L. Catapano - Olov Wiklund • \\ Yi Qian · Pratik Rane · Alyson Grove · Mona L. Martin
}

Received: July 23, 2019 / Published online: September 3, 2019

(C) The Author(s) 2019

\begin{abstract}
Introduction: Statin intolerance (SI) occurs in patients with dyslipidemia treated with statins. Statin-associated symptoms have been reported, but the overall patient experience is poorly understood. No instruments are available to
\end{abstract}

Enhanced Digital Features To view enhanced digital features for this article go to https://doi.org/10.6084/ m9.figshare.9632114.

Electronic supplementary material The online version of this article (https://doi.org/10.1007/s12325019-01073-7) contains supplementary material, which is available to authorized users.

M. Vrablik

Charles University and General University Hospital of Prague, Prague, Czech Republic

\section{A. L. Catapano}

University of Milan and Multimedica IRCCS, Milan, Italy

O. Wiklund

University of Gothenburg, Gothenburg, Sweden

Y. Qian · P. Rane

Amgen Inc., One Amgen Center Drive, Thousand

Oaks, CA, USA

\section{A. Grove}

RoundPeg Research, Oxford, UK

\section{L. Martin ( $\square)$}

Health Research Associates, Inc., 6505 216th St. SW, Suite 105, Mountlake Terrace, WA 98043, USA

e-mail: mona.martin@evidera.com collect this patient experience. Our aim is to develop a patient survey to define SI from the patient's perspective, inform clinical practice, and identify potential patient characteristics and barriers associated with discontinuing treatment when statin-related difficulties are encountered.

Methods: We conducted qualitative concept elicitation interviews with 65 patients across 12 European study sites. A semi-structured qualitative interview guide was developed based on literature review and clinician interviews. Concept elicitation interviews with patients were used to describe the patient experience and develop the conceptual framework for the survey.

Results: Symptoms experienced by patients included muscle and non-muscle-related pain and discomfort; other muscle-related symptoms; gastrointestinal, cardiovascular, cold-like, fatigue-related, and sensory and systems symptoms; mood changes; and cognitive and memory problems. Impacts included limitations on general physical functioning; physical activities; social functioning; emotional impacts; sleep disturbances; decreased productivity; and increased healthcare use. Conceptual framework elements to support survey goals include demographic and clinical characteristics, health information and beliefs, statin side-effect history, symptom severity, and impact severity.

Conclusions: Symptoms and impacts described by patients showed a wider range of symptoms 
and impacts than usually discussed clinically. The patient survey is designed to capture information from patients who experience difficulties with statin therapy and may be useful in identifying patients who are at higher risk for giving up or discontinuing their treatment.

Funding: Amgen Inc.

Keywords: Cardiology; Dyslipidemia; Lipidlowering therapy; Statin; Patient experience; Survey; Qualitative research

\section{INTRODUCTION}

Statins are a highly effective treatment for dyslipidemias [1]. They have been shown to reduce the risk of ischemic heart disease and stroke, and are one of the most commonly prescribed medications. In 2011, 13.0\% of Britain's population and Slovakia's population were prescribed a statin or statin-like drug, followed by Belgium (12.2\%), Norway (11.6\%), and Denmark (11.5\%), and with similar patterns throughout Europe [2]. In light of their prevalence, it is important to understand potential adverse drug reactions (ADRs) reported by patients that are associated with the use of statins.

While a number of studies have explored statin-associated adverse effects, there remains a lack of standard definitions of statin intolerance [3] or a clear clinical presentation of statin-related symptoms (e.g., myalgia) [4]. No biomarkers are currently available to detect the most common symptoms, statin-associated muscle symptoms [5] or additional symptoms that patients report. Differences in study design and nonuniformity of assessment tools have contributed to conflicting conclusions about the prevalence and severity of statin-associated ADRs [6]. This is further complicated by differing rates of reported adverse effects in clinical practice versus clinical trials, with clinical trials showing very low rates of ADRs [7]. Symptoms that have been reported to be associated with statin use are primarily muscle symptoms, but also include gastrointestinal, renal, and hepatic issues, mood and psychological disorders, and problems with cognition and memory $[8,9]$.
The symptoms and severity of statin intolerance vary from patient to patient and from medication to medication [10]. Notably, most patients who experience statin intolerance to one medication can tolerate subsequent trials of other statins [9].

We aimed to identify and understand the characteristics of statin intolerance from the perspective of the patient who is taking statins and experiencing difficulties. The overall objective of this work is to produce a patient survey to describe the burden of patient-perceived statin intolerance and potentially identify prospective patients who may have the same types of difficulties with statin treatment. This manuscript describes the qualitative study conducted to support the development of the content included in the patient survey.

\section{METHODS}

\section{Literature Review}

The first step in the development of the survey was to conduct a review of the literature to identify key elements of the patient experience that could be relevant in assessing symptom severity and life impacts in patients experiencing statin intolerance. We conducted a systematic search of articles published during 2001-2016 in PubMed. Additional articles were provided by the European Atherosclerosis Society. A total of 35 full articles that reported concepts of statin-related symptoms or impact were reviewed. Symptoms reported in the literature reviewed included muscle-related, gastrointestinal, renal/hepatic, mood/ psychological, and cognition symptoms and pain (Supplemental Table S1). Impacts identified from the literature review included limitations on physical functioning and daily activities, fatigue, sleep, and the patient-clinician relationship.

\section{Clinician Interviews}

A steering committee was formed to identify a sample of cardiovascular clinicians working in 
dyslipidemia across seven European countries: the Czech Republic, Italy, Norway, Slovakia, Spain, Sweden, and the UK. Sites were selected to represent different cultures and different types of practice settings. All clinicians had a current practice treating hyperlipidemia, experience using statins, and a concern about patients who experience difficulties with their statin treatment. Clinicians participated in two 60-min telephone interviews using a semistructured interview guide. Interviews were transcribed and used to summarize responses against key questions and topics.

Results from the literature review and clinician interviews were used to inform the content of the interview guide for patient concept elicitation interviews with patients.

\section{Patient Interviews}

Qualitative interviews were conducted with patients having difficulty with their prescribed statin treatment. These interviews were used to identify concepts relevant to the patient experience, and of interest to have included in the patient survey. Participants were identified and recruited by clinicians from 12 different study sites in the EU. These were two sites each in the Czech Republic, Italy, Spain, Slovakia, and Sweden and one site each in the UK and Norway. Ethics committee approval was obtained for each study site (Supplemental List S1), and all participants provided written informed consent prior to initiation of any study activity.

Eligible participants were $\geq 18$ years of age at screening, had been treated with $\geq 1$ statin within the 3 years prior to screening, and their prescribing physician had taken at least one of the following actions to address a side effect or symptom attributable (by either the patient or physician) to the prescribed statin therapy:

1. Statin therapy had been discontinued completely;

2. A switch to another statin occurred or prescribed dosage was lowered; and/or

3. Additional therapy (including nonprescribed medications, herbal medications, vitamins) was initiated.
Patients were excluded from the study if the action to change statin therapy was not based on a statin-related symptom (e.g., symptom related to a different medication or other preexisting condition), had a pre-existing neuromuscular condition (e.g., fibromyalgia, multiple sclerosis, muscular dystrophy), or untreated hypothyroidism.

Patients completed a demographic questionnaire during their enrollment visit and were scheduled for an individual face-to-face interview session. All interviews were conducted in person, in the native language of each country, and were audio-recorded and transcribed. During each interview, the semi-structured interview guide was used to elicit descriptions of the patient experience with statin-related difficulties. Interviews were all conducted in commercial research facilities and monitored by senior project staff. Simultaneous translation was used to provide the monitor with details of the interview in real time and to generate an English voice file for transcription. The transcripts were coded using Atlas-ti software by multiple coders to identify predominant statin-related symptoms and impact concepts.

To evaluate the consistency between coders and reliability of the coding process, a formal assessment of inter-rater agreement was conducted. Approximately 10\% of concept elicitation transcripts were independently dual-coded; the resulting transcript pairs were compared to evaluate any differences in the code assignment between the two coders. Upon completion of the coding process, saturation of concept (the point at which no new information is forthcoming from the concept elicitation interviews) was evaluated. Transcripts were ordered chronologically by the date conducted and divided into six groups. Each group of interviews was evaluated to identify the appearance of new information (new codes) and compared to the previous group to identify the point at which the interviews were no longer providing new information. This would indicate that no further information would be expected by continuing interviews with this population.

During the interview process, patients were asked to rate the severity of their symptoms on a scale of 0 (none) to 10 (extremely severe), and 
the bothersomeness of their symptoms on a scale of 0 (not bothersome at all) to 10 (extremely bothersome). Patients were also asked to rate the difficulty of the impacts of statin intolerance on their lives using a scale of 0 (not at all difficult to cope with) to 10 (extremely difficult to cope with).

\section{Survey Development}

The concept elicitation interview results were presented to the Steering Committee for discussion and decisions regarding the content that should be covered by the patient survey. Following these discussions, a conceptual framework was developed to reflect the proposed structure and subdomains, and a preliminary draft of the patient questionnaire was developed in English. Once the measure was approved by the team, the contents were translated into the appropriate language for each participating country. Country-specific information (such as the statin list that might be prescribed in each country) was reviewed by the site investigators, and the patient survey was prepared for the next step of assessment, in which the clarity and understandability of the items are checked with patients using cognitive interviews prior to the use of the survey for data collection.

\section{RESULTS}

\section{Clinician Interviews}

Fourteen clinicians participated in interviews, representing two hospital practices, five outpatient clinics, six specialty lipid clinics, and one private practice. Eight clinicians had $>20$ years of clinical experience. The frequency reported for patient visits for dyslipidemia ranged between 2 and 3 months up to 6 months or annually. The clinicians' perceptions of specific symptoms and impacts of statin intolerance are shown in Supplemental Table S2, and clinician quotations defining statin intolerance are reported in Supplemental Table S3. Most complaints received by clinicians were described by them as being about general activity limitation and reduced quality of life, tied largely to the types of activities that the patients felt were restricted (including choice of sports, everyday work, and walking for health and exercise). Two clinicians reported patient complaints that affected their sleep, and therefore had other quality of life repercussions on their subsequent days, such as chronic tiredness and fatigue.

When asked about determining whether or not a symptom was statin-related, 11 clinicians (79\%) indicated that they would base their decision on the typical practice of statin challenge, whereby the statin is discontinued and the patient is watched to see if the symptoms disappear, and 10 clinicians (71\%) indicated they would base their decisions on the results of medical tests (e.g., liver function, thyroid function, creatine kinase levels).

\section{Patients}

A total of 65 patients participated in concept elicitation interviews from the Czech Republic $(n=10)$, Italy $(n=10)$, Norway $(n=8)$, Slovakia $(n=10)$, Spain $(n=10)$, Sweden $(n=9)$, and the UK $(n=8)$. The mean age was 61.5 years [standard deviation (SD) 11.5] and slightly over half of the participants were male (54\%) (Table 1$)$. More than half of the patients $(60 \%)$ were no longer using a statin and several of these patients remarked during the interview process that they discontinued their statin treatment without consulting or informing their clinician.

\section{Results of Concept Elicitation Interviews}

A total of 5485 different quotations were coded as concepts from the 65 transcripts, and grouped by similarity of content. Symptoms expressed by patients included muscle-related pain and discomfort, non-muscle-related pain and discomfort, muscle-related symptoms that were different than pain, gastrointestinal symptoms, cardiovascular symptoms, cold-like symptoms, mood changes due to medication, cognitive and memory problems, fatigue-related symptoms, and sensory and systems symptoms (Table 2). Impacts expressed by patients included limitations on physical 
Table 1 Demographic and clinical characteristics of participating patients

\begin{tabular}{|c|c|c|c|c|c|c|c|c|}
\hline & $\begin{array}{l}\text { Czech } \\
\text { Republic } \\
(n=10)\end{array}$ & $\begin{array}{l}\text { Italy } \\
(n=10)\end{array}$ & $\begin{array}{l}\text { Norway } \\
(n=8)\end{array}$ & $\begin{array}{l}\text { Slovakia } \\
(n=10)\end{array}$ & $\begin{array}{l}\text { Spain } \\
(n=10)\end{array}$ & $\begin{array}{l}\text { Sweden } \\
(n=9)\end{array}$ & $\begin{array}{l}\text { United } \\
\text { Kingdom } \\
(n=8)\end{array}$ & $\begin{array}{l}\text { All } \\
\text { patients } \\
(n=65)\end{array}$ \\
\hline Age, mean years $(\mathrm{SD})$ & $\begin{array}{r}62.2 \\
(7.4)\end{array}$ & $\begin{array}{l}60.8 \\
(7.1)\end{array}$ & $\begin{array}{l}59.5 \\
(8.9)\end{array}$ & $\begin{array}{l}59.9 \\
\quad(14.9)\end{array}$ & $\begin{array}{l}63.5 \\
\quad(11.6)\end{array}$ & $\begin{array}{l}63.1 \\
\quad(17.9)\end{array}$ & NA & $\begin{array}{l}61.5 \\
\quad(11.5)\end{array}$ \\
\hline Sex, $n$ male $(\%)$ & $5(50)$ & $7(70)$ & $4(50)$ & $4(40)$ & $7(70)$ & $6(67)$ & $2(25)$ & $35(54)$ \\
\hline \multicolumn{9}{|l|}{ Highest education level, $n(\%)$} \\
\hline High school only & 0 & 0 & 0 & 0 & $1(10)$ & 0 & 0 & $1(2)$ \\
\hline Some college & $6(60)$ & $5(50)$ & $2(25)$ & $6(60)$ & $5(50)$ & $7(78)$ & 0 & $31(48)$ \\
\hline Bachelor's degree & $1(10)$ & $3(30)$ & $1(13)$ & $4(40)$ & $3(30)$ & $2(22)$ & $3(38)$ & $17(26)$ \\
\hline Graduate/professional school & $3(30)$ & $2(20)$ & $5(63)$ & 0 & $1(10)$ & 0 & $5(63)$ & $16(25)$ \\
\hline \multicolumn{9}{|l|}{ Employment status, $n(\%)$} \\
\hline Employed full-time & $4(40)$ & $5(50)$ & $3(38)$ & $3(30)$ & $4(40)$ & $2(22)$ & 0 & $21(32)$ \\
\hline Employed part-time & $1(10)$ & 0 & 0 & 0 & 0 & $1(11)$ & 0 & $2(3)$ \\
\hline Self-employed & $1(10)$ & $2(20)$ & 0 & $1(10)$ & $1(10)$ & 0 & $3(38)$ & $8(12)$ \\
\hline Retired & $4(40)$ & $3(30)$ & $4(50)$ & $6(60)$ & $4(40)$ & $6(67)$ & $5(63)$ & $32(49)$ \\
\hline Unable to work & 0 & 0 & $1(13)$ & 0 & $1(10)$ & 0 & 0 & $2(3)$ \\
\hline
\end{tabular}

Select comorbid conditions, $n(\%)$

\begin{tabular}{|c|c|c|c|c|c|c|c|c|}
\hline Cardiovascular disease & $5(50)$ & $6(60)$ & $3(38)$ & $4(40)$ & $6(60)$ & $6(67)$ & $4(50)$ & $34(52)$ \\
\hline Hypertension & $4(40)$ & $3(30)$ & $2(25)$ & $6(60)$ & $5(50)$ & $9(100)$ & $3(38)$ & $32(49)$ \\
\hline $\mathrm{FH}$ & $4(40)$ & $8(80)$ & $4(50)$ & $4(40)$ & $2(20)$ & 0 & $1(13)$ & $23(35)$ \\
\hline Diabetes & 0 & 0 & $1(13)$ & $4(40)$ & 0 & $2(22)$ & 0 & $7(11)$ \\
\hline Depression & 0 & 0 & 0 & 0 & 0 & 0 & 0 & 0 \\
\hline Sleep apnea & 0 & 0 & $3(38)$ & 0 & 0 & 0 & 0 & $3(5)$ \\
\hline $\begin{array}{l}\text { Time since first statin } \\
\text { prescription, mean years }(\mathrm{SD})\end{array}$ & $9.9(9.4)$ & $8.5(6.7)$ & $\begin{array}{l}14.9 \\
(13.9)\end{array}$ & $6.9(6.8)$ & $\begin{array}{l}9.9 \\
\quad(11.8)\end{array}$ & $\begin{array}{r}12.2 \\
(8.0)\end{array}$ & $10.1(8.4)$ & $\begin{array}{l}10.3 \\
(9.4)\end{array}$ \\
\hline $\begin{array}{l}\text { Satisfaction with current } \\
\text { treatment, }{ }^{a} \text { mean score }(\mathrm{SD}) \\
{[n]}\end{array}$ & $\begin{array}{l}5.2(4.9) \\
{[8]}\end{array}$ & $\begin{array}{c}3.9(3.9) \\
{[7]}\end{array}$ & $\begin{array}{l}5.3 \\
(4.5) \\
{[6]}\end{array}$ & $\begin{array}{c}4.9(3.9) \\
{[10]}\end{array}$ & $\begin{array}{l}6.2(4.4) \\
{[7]}\end{array}$ & $\begin{array}{c}10.0 \\
(0.0) \\
{[3]}\end{array}$ & $\begin{array}{l}4.0(\mathrm{NC}) \\
{[1]}\end{array}$ & $\begin{array}{c}5.4(4.1) \\
{[42]}\end{array}$ \\
\hline
\end{tabular}

Current number of statins, $n(\%)$

\begin{tabular}{lllllllll}
0 & $8(80)$ & $5(50)$ & $3(38)$ & $7(70)$ & $2(20)$ & $8(89)$ & $6(75)$ & $39(60)$ \\
1 & $2(20)$ & $3(30)$ & $2(25)$ & $3(30)$ & $6(60)$ & $1(11)$ & $2(25)$ & $19(29)$ \\
2 & 0 & $2(20)$ & $3(38)$ & 0 & $2(20)$ & 0 & 0 & $7(11)$ \\
\hline
\end{tabular}

$F H$ familial hypercholesterolemia, $[n]$ patients with data available, $N A$ not available, $N C$ not calculated, $S D$ standard deviation

a Satisfaction with treatment was scored on a numerical rating scale from 0 (not satisfied at all) to 10 (extremely satisfied) 
Table 2 Statin intolerance symptom code frequencies

\begin{tabular}{|c|c|c|c|c|}
\hline Symptoms & $\begin{array}{l}\text { No. patient language } \\
\text { expressions within } \\
\text { concept }\end{array}$ & $\begin{array}{l}\% \text { of } 2069 \\
\text { symptom } \\
\text { expressions (\%) }\end{array}$ & $\begin{array}{l}\text { No. transcripts } \\
\text { contributing to concept } \\
\text { expression }\end{array}$ & $\begin{array}{l}\% \text { of } 65 \text { transcripts } \\
\text { contributing }(\%)\end{array}$ \\
\hline $\begin{array}{l}\text { Non-muscle-related } \\
\text { pain and } \\
\text { discomfort }\end{array}$ & 289 & 14 & & \\
\hline $\begin{array}{l}\text { Arthritis and bone } \\
\text { symptoms }\end{array}$ & 181 & 8.7 & 33 & 50.8 \\
\hline $\begin{array}{l}\text { Bone and joint } \\
\text { weakness }\end{array}$ & 5 & 0.2 & 3 & 4.6 \\
\hline $\begin{array}{l}\text { Burning and } \\
\text { stinging }\end{array}$ & 21 & 1.0 & 6 & 9.2 \\
\hline Headache & 23 & 1.1 & 7 & 10.8 \\
\hline $\begin{array}{l}\text { Non-muscle neck } \\
\text { and back pain }\end{array}$ & 15 & 0.7 & 7 & 10.8 \\
\hline $\begin{array}{l}\text { Non-muscle pain } \\
\text { or discomfort }\end{array}$ & 44 & 2.1 & 11 & 16.9 \\
\hline $\begin{array}{l}\text { Muscle-related pain } \\
\text { or discomfort }\end{array}$ & 574 & 28 & & \\
\hline Muscle ache & 106 & 5.1 & 33 & 50.8 \\
\hline Muscle burning & 7 & 0.3 & 1 & 1.5 \\
\hline Cramps & 152 & 7.3 & 32 & 49.2 \\
\hline $\begin{array}{l}\text { Muscle pulls and } \\
\text { tears }\end{array}$ & 6 & 0.3 & 4 & 6.2 \\
\hline $\begin{array}{l}\text { Soreness and } \\
\text { tenderness }\end{array}$ & 47 & 2.3 & 23 & 35.4 \\
\hline $\begin{array}{l}\text { Muscle pain or } \\
\text { discomfort }\end{array}$ & 256 & 12.4 & 50 & 76.9 \\
\hline $\begin{array}{l}\text { Non-pain muscle- } \\
\text { related symptoms }\end{array}$ & 279 & 13 & & \\
\hline Muscle weakness & 116 & 5.6 & 32 & 49.2 \\
\hline $\begin{array}{l}\text { Muscle stiffness } \\
\text { and tightness }\end{array}$ & 90 & 4.3 & 33 & 50.8 \\
\hline Muscle tingling & 27 & 1.3 & 8 & 12.3 \\
\hline Muscle loss & 21 & 1.0 & 5 & 7.7 \\
\hline Muscle lump & 4 & 0.2 & 1 & 1.5 \\
\hline
\end{tabular}


Table 2 continued

\begin{tabular}{|c|c|c|c|c|}
\hline Symptoms & $\begin{array}{l}\text { No. patient language } \\
\text { expressions within } \\
\text { concept }\end{array}$ & $\begin{array}{l}\text { \% of } 2069 \\
\text { symptom } \\
\text { expressions (\%) }\end{array}$ & $\begin{array}{l}\text { No. transcripts } \\
\text { contributing to concept } \\
\text { expression }\end{array}$ & $\begin{array}{l}\% \text { of } 65 \text { transcripts } \\
\text { contributing }(\%)\end{array}$ \\
\hline $\begin{array}{l}\text { Other muscle } \\
\text { issues }^{\mathrm{a}}\end{array}$ & 21 & 1.0 & 8 & 12.3 \\
\hline $\begin{array}{l}\text { Gastrointestinal } \\
\text { symptoms }\end{array}$ & 115 & 6 & & \\
\hline $\begin{array}{l}\text { Abdominal } \\
\text { discomfort }\end{array}$ & 8 & 0.4 & 3 & 4.6 \\
\hline $\begin{array}{l}\text { Abdominal pain or } \\
\text { cramps }\end{array}$ & 29 & 1.4 & 11 & 16.9 \\
\hline Acid reflux & 4 & 0.2 & 2 & 3.1 \\
\hline Bloating & 4 & 0.2 & 2 & 3.1 \\
\hline Constipation & 8 & 0.4 & 4 & 6.2 \\
\hline Diarrhea & 32 & 1.5 & 11 & 16.9 \\
\hline Nausea & 23 & 1.1 & 8 & 12.3 \\
\hline Stool changes & 4 & 0.2 & 1 & 1.5 \\
\hline Vomiting & 3 & 0.1 & 1 & 1.5 \\
\hline $\begin{array}{l}\text { Cardiovascular } \\
\text { symptoms }\end{array}$ & 28 & 1 & & \\
\hline Arrhythmia & 4 & 0.2 & 1 & 1.5 \\
\hline $\begin{array}{l}\text { Blood pressure } \\
\text { changes }\end{array}$ & 6 & 0.3 & 3 & 4.6 \\
\hline Chest pain & 4 & 0.2 & 2 & 3.1 \\
\hline $\begin{array}{l}\text { Difficulty } \\
\text { breathing }\end{array}$ & 9 & 0.4 & 5 & 7.7 \\
\hline Heart palpitations & 3 & 0.1 & 1 & 1.5 \\
\hline Increased heart rate & 2 & 0.1 & 1 & 1.5 \\
\hline Cold-like symptoms & 127 & 6 & & \\
\hline Chills & 7 & 0.3 & 6 & 9.2 \\
\hline Coughing & 3 & 0.1 & 2 & 3.1 \\
\hline Dizziness & 32 & 1.5 & 12 & 18.5 \\
\hline Feels hot & 12 & 0.6 & 3 & 4.6 \\
\hline Feels sick or poorly & 44 & 2.1 & 18 & 27.7 \\
\hline $\begin{array}{l}\text { Runny or stuffy } \\
\text { nose }\end{array}$ & 7 & 0.3 & 6 & 9.2 \\
\hline
\end{tabular}


Table 2 continued

\begin{tabular}{|c|c|c|c|c|}
\hline Symptoms & $\begin{array}{l}\text { No. patient language } \\
\text { expressions within } \\
\text { concept }\end{array}$ & $\begin{array}{l}\% \text { of } 2069 \\
\text { symptom } \\
\text { expressions (\%) }\end{array}$ & $\begin{array}{l}\text { No. transcripts } \\
\text { contributing to concept } \\
\text { expression }\end{array}$ & $\begin{array}{l}\% \text { of } 65 \text { transcripts } \\
\text { contributing }(\%)\end{array}$ \\
\hline Sneezing & 2 & 0.1 & 2 & 3.1 \\
\hline Throat symptoms & 19 & 0.9 & 9 & 13.8 \\
\hline $\begin{array}{l}\text { Other cold-like } \\
\text { symptoms }\end{array}$ & 1 & $<0.1$ & 1 & 1.5 \\
\hline $\begin{array}{l}\text { Mood changes due } \\
\text { to medication }\end{array}$ & 94 & 5 & & \\
\hline Anxiety & 28 & 1.4 & 10 & 15.4 \\
\hline Apathy & 4 & 0.2 & 1 & 1.5 \\
\hline Depression & 45 & 2.2 & 16 & 24.6 \\
\hline $\begin{array}{l}\text { Hostility or } \\
\text { agitation }\end{array}$ & 11 & 0.5 & 5 & 7.7 \\
\hline Lack of confidence & 5 & 0.2 & 1 & 1.5 \\
\hline $\begin{array}{l}\text { Other mood } \\
\text { changes }^{c}\end{array}$ & 1 & $<0.1$ & 1 & 1.5 \\
\hline $\begin{array}{l}\text { Cognitive and } \\
\text { memory problems }\end{array}$ & 108 & 5 & & \\
\hline $\begin{array}{l}\text { Cognitive } \\
\text { problems }\end{array}$ & 44 & 2.1 & 14 & 21.5 \\
\hline Memory problems & 64 & 3.1 & 16 & 24.6 \\
\hline $\begin{array}{l}\text { Fatigue-related } \\
\text { symptoms }\end{array}$ & 299 & 14 & & \\
\hline Exhaustion & 45 & 2.2 & 21 & 32.3 \\
\hline Fatigue & 50 & 2.4 & 22 & 33.8 \\
\hline Lack of energy & 76 & 3.7 & 31 & 47.7 \\
\hline Tiredness & 118 & 5.7 & 36 & 55.4 \\
\hline Weakness & 10 & 0.5 & 6 & 9.2 \\
\hline $\begin{array}{l}\text { Sensory and systems } \\
\text { problems }\end{array}$ & 67 & 3 & & \\
\hline Eye symptoms & 4 & 0.2 & 2 & 3.1 \\
\hline Ear symptoms & 5 & 0.2 & 3 & 4.6 \\
\hline Mouth symptoms & 3 & 0.1 & 1 & 1.5 \\
\hline $\begin{array}{l}\text { Skin and hair } \\
\text { symptoms }\end{array}$ & 55 & 2.7 & 12 & 18.5 \\
\hline
\end{tabular}


Table 2 continued

\begin{tabular}{|c|c|c|c|c|}
\hline Symptoms & $\begin{array}{l}\text { No. patient language } \\
\text { expressions within } \\
\text { concept }\end{array}$ & $\begin{array}{l}\text { \% of } 2069 \\
\text { symptom } \\
\text { expressions (\%) }\end{array}$ & $\begin{array}{l}\text { No. transcripts } \\
\text { contributing to concept } \\
\text { expression }\end{array}$ & $\begin{array}{l}\% \text { of } 65 \text { transcripts } \\
\text { contributing }(\%)\end{array}$ \\
\hline $\begin{array}{l}\text { Additional } \\
\text { symptoms }\end{array}$ & 89 & 4 & & \\
\hline Allergy & 1 & $<0.1$ & 1 & 1.5 \\
\hline High blood sugar & 1 & $<0.1$ & 1 & 1.5 \\
\hline Infections & 1 & $<0.1$ & 1 & 1.5 \\
\hline Kidney problems & 10 & 0.5 & 7 & 10.8 \\
\hline Liver problems & 20 & 1.0 & 7 & 10.8 \\
\hline Sweating & 8 & 0.4 & 5 & 7.7 \\
\hline $\begin{array}{l}\text { Swelling or } \\
\text { inflammation }\end{array}$ & 26 & 1.3 & 11 & 16.9 \\
\hline $\begin{array}{l}\text { Uncoordinated } \\
\text { movement }\end{array}$ & 4 & 0.2 & 1 & 1.5 \\
\hline $\begin{array}{l}\text { Weight-related } \\
\text { symptoms }\end{array}$ & 18 & 0.9 & 5 & 7.7 \\
\hline
\end{tabular}

a Other muscle issues included congestion, creatine kinase, heat, and spine blocked

b Other cold-like symptoms included heavy head

c Other mood changes included suicidal thoughts

functioning in general, physical activities, and social functioning. Patients also noted emotional impacts, sleep disturbances, impacts on productivity, and increased use of healthcare (Table 3).

While a few new codes were still coming forward in the last transcript group for the saturation analysis, it was clear from the content that the concepts being expressed were not new concepts but the use of different language to describe the same concept, and were basically reflecting the variation between language use in the countries. Therefore, the developers felt confident that all relevant concepts from this group of patients were registered for consideration. Inter-rater agreement ranged between 94.0 to $98.5 \%$ for the assignment of codes.

Patients were asked to rate the severity and bothersomeness of statin-related symptoms (Table 4). Notably, some symptoms with higher severity ratings were associated with low levels of bothersomeness (e.g., cold-like symptoms of chills), and conversely, some symptoms with lower severity ratings had higher bothersomeness ratings (e.g., constipation). Patients were also asked to rate the difficulty of statin-related impacts (Table 5). Based on these ratings, impacts on relationships had the highest (i.e., greatest difficulty) ratings. The severity and bothersomeness ratings were used in conjunction with the coded transcript data to determine the most relevant content to include in the design of the survey.

\section{Conceptual Framework for Statin Intolerance Patient Survey}

The goals of the survey are to help describe statin intolerance from the patient perspective, inform clinical practice around statin intolerance, and to identify potential risk characteristics or barriers that may result in patients giving 
Table 3 Statin intolerance impact code frequencies

\begin{tabular}{|c|c|c|c|c|}
\hline Concept description & $\begin{array}{l}\text { No. patient language } \\
\text { expressions within } \\
\text { concept }\end{array}$ & $\begin{array}{l}\% \text { of } 1275 \\
\text { impact } \\
\text { expressions }\end{array}$ & $\begin{array}{l}\text { No. transcripts } \\
\text { contributing to concept } \\
\text { expression }\end{array}$ & $\begin{array}{l}\% \text { of } 65 \\
\text { transcripts } \\
\text { contributing }\end{array}$ \\
\hline $\begin{array}{l}\text { Impacts on physical } \\
\text { activities and } \\
\text { functioning }\end{array}$ & 461 & 36 & & \\
\hline Falls & 6 & 0.5 & 2 & 3.1 \\
\hline Hand function limited & 9 & 0.7 & 6 & 9.2 \\
\hline Have to rest more & 57 & 4.5 & 30 & 46.2 \\
\hline $\begin{array}{l}\text { Physical activities } \\
\text { limited }\end{array}$ & 183 & 14.4 & 50 & 76.9 \\
\hline $\begin{array}{l}\text { Physical functioning } \\
\text { limited }\end{array}$ & 206 & 16.2 & 48 & 73.8 \\
\hline $\begin{array}{l}\text { Impacts on social } \\
\text { functioning }\end{array}$ & 170 & 13 & & \\
\hline Activity harder & 5 & 0.4 & 4 & 6.2 \\
\hline $\begin{array}{l}\text { Altered close } \\
\text { relationships }\end{array}$ & 20 & 1.6 & 11 & 16.9 \\
\hline $\begin{array}{l}\text { Altered relationships } \\
\text { with friends }\end{array}$ & 8 & 0.6 & 7 & 10.8 \\
\hline $\begin{array}{l}\text { General functioning } \\
\text { limited }\end{array}$ & 60 & 4.7 & 35 & 53.8 \\
\hline Lifestyle restrictions & 31 & 2.4 & 19 & 29.2 \\
\hline Sexual activity & 4 & 0.3 & 2 & 3.1 \\
\hline $\begin{array}{l}\text { Social activities } \\
\text { affected }\end{array}$ & 42 & 3.3 & 26 & 40.0 \\
\hline Emotional Impacts & 283 & 22 & & \\
\hline Anxiety & 80 & 6.3 & 28 & 43.1 \\
\hline Apathy & 5 & 0.4 & 2 & 3.1 \\
\hline Depression & 51 & 4.0 & 22 & 33.8 \\
\hline Frustration & 27 & 2.1 & 14 & 21.5 \\
\hline Hopelessness & 5 & 0.4 & 3 & 4.6 \\
\hline Hostility or aggression & 38 & 3.0 & 19 & 29.2 \\
\hline Lack of confidence & 12 & 0.9 & 10 & 15.4 \\
\hline Mental Tiredness & 5 & 0.4 & 2 & 3.1 \\
\hline Stress & 16 & 1.3 & 10 & 15.4 \\
\hline
\end{tabular}


Table 3 continued

\begin{tabular}{|c|c|c|c|c|}
\hline Concept description & $\begin{array}{l}\text { No. patient language } \\
\text { expressions within } \\
\text { concept }\end{array}$ & $\begin{array}{l}\text { \% of } 1275 \\
\text { impact } \\
\text { expressions }\end{array}$ & $\begin{array}{l}\text { No. transcripts } \\
\text { contributing to concept } \\
\text { expression }\end{array}$ & $\begin{array}{l}\text { \% of } 65 \\
\text { transcripts } \\
\text { contributing }\end{array}$ \\
\hline Worry & 44 & 3.5 & 20 & 30.8 \\
\hline Sleep disturbances & 124 & 10 & & \\
\hline Difficulty falling asleep & 30 & 2.4 & 22 & 33.8 \\
\hline $\begin{array}{l}\text { Difficulty staying } \\
\text { asleep }\end{array}$ & 49 & 3.8 & 25 & 38.5 \\
\hline Poor quality sleep & 45 & 3.5 & 23 & 35.4 \\
\hline Impacts on productivity & 132 & 10 & & \\
\hline Diminished work & 25 & 2.0 & 15 & 23.1 \\
\hline Limitations to chores & 34 & 2.7 & 17 & 26.2 \\
\hline $\begin{array}{l}\text { Poor performance or } \\
\text { productivity }\end{array}$ & 43 & 3.4 & 20 & 30.8 \\
\hline Trouble reading & 5 & 0.4 & 3 & 4.6 \\
\hline Work more difficult & 25 & 2.0 & 17 & 26.2 \\
\hline Use of healthcare & 58 & 5 & & \\
\hline Increased doctor visits & 42 & 3.3 & 33 & 50.8 \\
\hline $\begin{array}{l}\text { Increased } \\
\text { hospitalization }\end{array}$ & 12 & 0.9 & 9 & 13.8 \\
\hline Treatment burden & 4 & 0.3 & 4 & 6.2 \\
\hline Additional impacts & 47 & 4 & & \\
\hline Altered attire & 9 & 0.7 & 3 & 4.6 \\
\hline Dietary changes & 3 & 0.2 & 2 & 3.1 \\
\hline Financial burden & 2 & 0.2 & 2 & 3.1 \\
\hline Increased dependence & 2 & 0.2 & 2 & 3.1 \\
\hline Overall quality of life & 31 & 2.4 & 22 & 33.8 \\
\hline
\end{tabular}

up and discontinuing their statin treatment. Six key elements were identified for the conceptual framework for the survey (Table 6): (1) respondent demographics; (2) clinical characteristics;
(3) health information and beliefs; (4) statin side effect history; (5) symptom severity; and (6) impact severity. 
Table 4 Statin intolerance symptom severity and bothersomeness ratings

\begin{tabular}{llll}
\hline Symptoms & All patients $(n=65)$ \\
& $\frac{\text { Symptom severity }}{n \quad \text { Mean score (SD) [range] }}$ & & $\frac{\text { Symptom bothersomeness }}{n}$ \\
\hline
\end{tabular}

Non-muscle-related pain and discomfort

Arthritis and bone symptoms

Bone or joint ache

Bone or joint pain

Stiff or cracking joints

Soreness

Tenderness

Bone or joint weakness

Joint weakness

Burning and stinging

Burning

Stinging

Headache

Non-muscle neck or back pain

Non-muscle pain or discomfort

Muscle-related pain or discomfort

Muscle ache

Muscle burning

Muscle cramps

Muscle tears

Soreness and tenderness

\section{Soreness}

Tenderness

Muscle pain or discomfort

Non-pain muscle-related symptoms

Muscle weakness

Muscle fatigue

Muscle heaviness

Muscle weakness

Muscle stiffness

Muscle tingling

$$
\begin{array}{rl}
10 & 7.7(0.9)[6-9] \\
3 & 5.7(2.9)[4-9] \\
3 & 6.7(3.5)[3-10] \\
5 & 7.8(2.0)[6-10] \\
3 & 7.0(1.7)[5-8]
\end{array}
$$

$0 \quad$ NA

$$
\begin{array}{rl}
2 & 8.5(2.1)[7-10] \\
0 & \text { NA } \\
4 & 7.3(2.1)[5-10] \\
0 & \text { NA } \\
21 & 7.4(2.0)[2-10]
\end{array}
$$

$$
\begin{array}{rl}
23 & 7.4(2.1)[3-10] \\
0 & \text { NA } \\
25 & 7.4(2.2)[2-10] \\
1 & 10.0
\end{array}
$$$$
47.8 \text { (2.1) [6-10] }
$$$$
267.7(2.1)[3-10]
$$$$
210.0(0) \text { [10] }
$$$$
77.1(3.0)[3-10]
$$$$
7 \quad 7.7 \text { (1.3) [6-10] }
$$

18.0

$$
\begin{array}{rl}
1 & 9.0 \\
1 & 8.0 \\
5 & 6.2(2.2)[5-10] \\
6 & 8.2(3.0)[3-10] \\
14 & 7.6(1.6)[5-10]
\end{array}
$$

$$
\begin{array}{rrrr}
12 & 8.1(1.8)[5-10] & 14 & 6.8(1.9)[4-10] \\
8 & 8.3(1.7)[5-10] & 7 & 7.0(1.9)[4-10] \\
39 & 7.2(2.3)[3-10] & 26 & 7.1(2.4)[1-10]
\end{array}
$$$$
28 \quad 6.4(2.0)[2-10]
$$$$
17.0
$$$$
267.0(2.5)[2-10]
$$$$
29.0(1.4)[8-10]
$$

$$
\begin{array}{rl}
13 & 7.1(2.2)[4-10] \\
18 & 6.9(2.2)[2-10] \\
26 & 7.0(2.0)[2-10] \\
28 & 6.4(2.2)[1-10] \\
5 & 6.2(1.3)[5-8]
\end{array}
$$


Table 4 continued

\begin{tabular}{|c|c|c|c|c|}
\hline \multirow[t]{3}{*}{ Symptoms } & \multicolumn{4}{|c|}{ All patients $(n=65)$} \\
\hline & \multicolumn{2}{|c|}{ Symptom severity } & \multicolumn{2}{|c|}{ Symptom bothersomeness } \\
\hline & $\bar{n}$ & Mean score (SD) [range] & $\bar{n}$ & $\overline{\text { Mean score }(\mathrm{SD}) \text { [range] }}$ \\
\hline \multicolumn{5}{|l|}{ Muscle loss } \\
\hline Lack of tone & 1 & 5.0 & 1 & 7.0 \\
\hline Muscle loss & 1 & 3.5 & 4 & $6.0(1.8)[4-8]$ \\
\hline Muscle wasting & 0 & NA & 1 & 10.0 \\
\hline Muscle lump & 0 & NA & 1 & 6.0 \\
\hline Warm/heat in upper leg; blocking spine chest part & 2 & $8.8(1.8)[7.5-10]$ & 2 & $8.5(2.1)[7-10]$ \\
\hline \multicolumn{5}{|l|}{ Gastrointestinal symptoms } \\
\hline \multicolumn{5}{|l|}{ Abdominal discomfort } \\
\hline Poor digestion & 1 & 8.0 & 1 & 8.0 \\
\hline Abdominal pain or cramps & 6 & $8.0(1.4)[6-10]$ & 8 & $6.4(2.3)[3-9]$ \\
\hline Acid reflux & 1 & 10.0 & 1 & 10.0 \\
\hline Bloating & 2 & $7.5(0.7)[7-8]$ & 1 & 7.0 \\
\hline Constipation & 2 & $5.0(2.8)[3-7]$ & 3 & $8.0(2.0)[6-10]$ \\
\hline Diarrhea & 9 & $7.3(1.9)[4-9]$ & 11 & $6.1(2.8)[1-10]$ \\
\hline Nausea & 5 & $7.2(2.6)[4-10]$ & 6 & $6.7(3.4)[2-10]$ \\
\hline Stool changes & 0 & NA & 1 & 7.0 \\
\hline Vomiting & 4 & $6.8(2.8)[4-10]$ & 2 & $7.5(3.5)[5-10]$ \\
\hline \multicolumn{5}{|l|}{ Cardiovascular symptoms } \\
\hline Difficulty breathing & 0 & NA & 4 & $9.3(1.0)[8-10]$ \\
\hline Heart palpitations & 1 & 8.5 & 2 & $7.0(2.8)[5-9]$ \\
\hline Increased heart rate & 1 & 5.0 & 0 & NA \\
\hline \multicolumn{5}{|l|}{ Cold-like symptoms } \\
\hline Chills & 2 & $7.0(1.4)[6-8]$ & 3 & $5.3(2.1)[3-7]$ \\
\hline \multicolumn{5}{|l|}{ Dizziness } \\
\hline Dizziness & 2 & $8.5(0.7)[8-9]$ & 2 & $9.0(1.4)[8-10]$ \\
\hline Feels faint & 2 & $9.5(0.7)[9-10]$ & 1 & 10.0 \\
\hline Feels sick or poorly & 6 & $6.8(1.8)[5-10]$ & 8 & $6.9(2.5)[2-10]$ \\
\hline Feels hot & 2 & $7.0(1.4)[6-8]$ & 2 & $7.5(2.1)[6-9]$ \\
\hline Runny or stuffy nose & 3 & $8.7(1.2)[8-10]$ & 4 & $5.3(3.0)[2-9]$ \\
\hline Sneezing & 1 & 8.0 & 4 & $4.0(0.8)[3-5]$ \\
\hline
\end{tabular}


Table 4 continued Symptoms

All patients $(n=65)$

Symptom severity

$\bar{n} \quad$ Mean score (SD) [range]

Symptom bothersomeness

$\bar{n}$ Mean score (SD) [range]

Throat symptoms

Sore throat

$5 \quad 6.5(2.8)[3-9.5]$

$8 \quad 5.5(2.7)[1-9]$

Mood changes due to medication

Anxiety

Anxiety

Nervous

$127.8(2.8)[1-10]$

$0 \quad$ NA

$128.4(1.2)[6-10]$

18.0

Depression

Depression

Low mood

Sadness

Mood changes

Hostility or agitation

$69.2(1.3)[7-10]$

$7 \quad 7.9(1.7)[5-10]$

15.0

16.0

$29.5(0.7)[9-10]$

37.7 (1.5) [6-9]

0 NA

17.0

$58.4(2.2)[6-10]$

$6 \quad 6.8(2.8)[3-10]$

Cognitive and memory problems

Cognitive symptoms

Fuzzy thoughts

Difficulty concentrating

$0 \quad \mathrm{NA}$

$28.5(2.1)[7-10]$

19.0

Memory problems

$$
\begin{aligned}
& \text { Amnesia } \\
& \text { Memory problems }
\end{aligned}
$$

$210.0(0.0)[10]$

$0 \quad$ NA

$107.2(2.3)[4-10]$

$10 \quad 8.2(2.1)[4-10]$

Fatigue-related symptoms

Fatigue

$0 \quad \mathrm{NA}$

$15 \quad 7.1(1.9)[3-10]$

Exhaustion

$17 \quad 7.3(1.8)[5-10]$

$227.5(2.0)[2-10]$

Lack of energy

$247.1(2.2)[3-10]$

$267.4(2.1)[2-10]$

Tiredness

$24 \quad 6.7(2.3)[3-10]$

Sensory and systems problems

Skin and hair symptoms

Bruising

Eczema

Hair loss

Rash

Sensitive skin
$\begin{array}{ll}1 & 2.0\end{array}$
$0 \quad$ NA
$29.0(0.0)$ [9]
$28.5(2.1)[7-10]$
$0 \quad \mathrm{NA}$

$1 \quad 6.0$

16.0

$210.0(0.0)[10]$

$29.5(0.7)[9-10]$ 
Table 4 continued

\begin{tabular}{|c|c|c|c|c|}
\hline \multirow[t]{3}{*}{ Symptoms } & \multicolumn{4}{|c|}{ All patients $(n=65)$} \\
\hline & \multicolumn{2}{|c|}{ Symptom severity } & \multicolumn{2}{|c|}{ Symptom bothersomeness } \\
\hline & $n$ & Mean score (SD) [range] & $n$ & Mean score (SD) [range] \\
\hline Skin ulcers & 1 & 6.0 & 1 & 10.0 \\
\hline \multicolumn{5}{|l|}{ Additional symptoms } \\
\hline \multicolumn{5}{|l|}{ Kidney problems } \\
\hline Frequent urination & 0 & NA & 2 & $7.0(4.2)[4-10]$ \\
\hline Liver problems & 1 & 8.0 & 2 & $7.0(4.2)[4-10]$ \\
\hline Sweating & 0 & NA & 2 & $9.0(1.4)[8-10]$ \\
\hline \multicolumn{5}{|c|}{ Swelling or inflammation } \\
\hline Swelling & 2 & $9.5(0.7)[9-10]$ & 4 & $8.3(1.7)[6-10]$ \\
\hline \multicolumn{5}{|c|}{ Weight-related symptoms } \\
\hline Fat loss & 0 & NA & 1 & 0.0 \\
\hline Weight gain & 1 & 10.0 & 1 & 10.0 \\
\hline
\end{tabular}

$n$ number of patients rating symptom, $N A$ not applicable, $S D$ standard deviation

Table 5 Statin intolerance impact difficulty ratings

Concept description

Impacts on physical activities and functioning

Falls

Have to rest more

Physical activities limited

Physical functioning limited

Impacts on social functioning

Altered close relationships

Altered relationships with friends

Sexual activity

Social activities affected

Lifestyle restrictions

General functioning limited

Activity harder

Emotional impacts

Anxiety

Mentally tired

Stress

\begin{tabular}{ll} 
All patients $(n=65)$ & \\
\hline$n$ & Mean score (SD) [range]
\end{tabular}

1

3.0

17

32

26

$6.8(1.6)[4-9]$

$6.5(2.6)[1-10]$

$7.1(2.5)[1-10]$

5

2

$8.4(1.7)[6-10]$

$9.0(1.4)[8-10]$

1

8.0

16

$5.7(2.3)[1-8]$

15

12

$7.2(1.6)[5-10]$

$6.0(2.0)[3-9]$

1

6.0

13

$6.8(2.6)$ [3-10]

1

10.0

1

6.0 
Table 5 continued

\begin{tabular}{|c|c|c|}
\hline \multirow[t]{2}{*}{ Concept description } & \multicolumn{2}{|c|}{ All patients $(n=65)$} \\
\hline & $n$ & Mean score (SD) [range] \\
\hline Worry & 16 & $6.9(2.1)[2-10]$ \\
\hline \multicolumn{3}{|l|}{ Depression } \\
\hline Depression & 8 & $7.3(1.7)[5-10]$ \\
\hline Low mood & 3 & $5.7(2.5)[3-8]$ \\
\hline Sadness & 15 & $6.0(2.5)[3-10]$ \\
\hline \multicolumn{3}{|l|}{ Frustration } \\
\hline Annoyance & 1 & 6.0 \\
\hline Frustration & 11 & $7.9(1.8)[4-10]$ \\
\hline \multicolumn{3}{|l|}{ Hostility or agitation } \\
\hline Anger & 4 & $6.8(2.1)[4-9]$ \\
\hline Irritability & 1 & 8.0 \\
\hline \multicolumn{3}{|l|}{ Lack of confidence } \\
\hline Embarrassment & 6 & $6.2(3.5)[1-10]$ \\
\hline Self-deprecation & 1 & 8.0 \\
\hline \multicolumn{3}{|l|}{ Sleep disturbances } \\
\hline Difficulty falling asleep & 10 & $6.6(2.9)[3-10]$ \\
\hline Difficulty staying asleep & 6 & $8.0(1.7)[5-10]$ \\
\hline Poor quality sleep & 23 & $7.3(2.0)[3-10]$ \\
\hline \multicolumn{3}{|l|}{ Impacts on productivity } \\
\hline Diminished work & 10 & $6.8(3.1)[0-10]$ \\
\hline Limitations to chores & 8 & $7.4(1.8)[5-10]$ \\
\hline Poor performance or productivity & 17 & $6.6(3.0)[0-10]$ \\
\hline Work more difficult & 1 & 5.0 \\
\hline \multicolumn{3}{|l|}{ Use of healthcare } \\
\hline Increased doctor visits & 23 & $5.8(2.9)[0-10]$ \\
\hline Increased hospitalization & 4 & $7.8(2.6)[4-10]$ \\
\hline \multicolumn{3}{|l|}{ Additional impacts } \\
\hline Altered attire & 1 & 10.0 \\
\hline Increased dependence & 1 & 8.0 \\
\hline Overall quality of life & 1 & 8.0 \\
\hline
\end{tabular}

$n$ number of patients rating symptom, $N A$ not applicable, $S D$ standard deviation 
Table 6 Conceptual framework for statin intolerance patient survey

\begin{tabular}{|c|c|}
\hline Concept & Items for survey \\
\hline \multirow[t]{5}{*}{ Respondent demographics } & Age \\
\hline & Sex \\
\hline & Marital status \\
\hline & Education level \\
\hline & Employment status \\
\hline \multirow[t]{14}{*}{ Clinical characteristics } & Statin intolerant group $(\mathrm{Y} / \mathrm{N})$ \\
\hline & Self-reported health status \\
\hline & Comorbid conditions \\
\hline & Perception of risk for myocardial infarction \\
\hline & Diagnosis of familial hypercholesterolemia \\
\hline & Family history of medical conditions \\
\hline & History of non-statin side effects \\
\hline & Knowing others who have discontinued a statin \\
\hline & Current prescriptions per day \\
\hline & Current over-the-counter medications per day \\
\hline & Activity level \\
\hline & Alcohol \\
\hline & Smoking \\
\hline & Patient Health Questionnaire (PHQ-4) \\
\hline \multirow[t]{11}{*}{ Health information and beliefs } & Comfort level with Dr. \\
\hline & Quality of communication with Dr. \\
\hline & Type of Dr. providing prescribing statin \\
\hline & Feels listened to by Dr. \\
\hline & Willingness to try another statin \\
\hline & Level of involvement with medical care decisions \\
\hline & Satisfaction with information from Dr. \\
\hline & Information sources (frequency of use, level of trust) \\
\hline & Reason for taking a statin \\
\hline & Reason for not taking a statin \\
\hline & Cholesterol under control or not \\
\hline
\end{tabular}


Table 6 continued

\begin{tabular}{|c|c|}
\hline Concept & Items for survey \\
\hline \multirow[t]{12}{*}{ Statin side effect history } & Statins taken \\
\hline & Current use of a statin or not \\
\hline & Side effects with previous statin \\
\hline & Type of side effects \\
\hline & Side effects with current or most recent statin \\
\hline & Time to onset of side effects \\
\hline & Side effect interference with activities \\
\hline & Type of changes made to address side effects \\
\hline & Improvements after changes made \\
\hline & Time to improvement \\
\hline & Timing of most recent side effect experience \\
\hline & Current over-the-counter supplement \\
\hline \multirow[t]{15}{*}{ Symptom severity } & Muscle aches \\
\hline & Muscle cramps \\
\hline & Muscle pain \\
\hline & Muscle soreness \\
\hline & Muscle stiffness \\
\hline & Muscle weakness \\
\hline & Bone and joint pain \\
\hline & Abdominal pain \\
\hline & Lack of energy \\
\hline & Tires easily \\
\hline & Exhaustion \\
\hline & Fatigue \\
\hline & Memory problems \\
\hline & Irritability \\
\hline & Frustration \\
\hline
\end{tabular}


Table 6 continued

\begin{tabular}{ll}
\hline Concept & Items for survey \\
\hline Impact severity & Needing to rest more \\
& Reduced ability to be physically active \\
& Limited social activities \\
& Increased office visits \\
& Reduced productivity or performance \\
& Reduced ability to exercise \\
& Trouble getting good quality sleep \\
\hline
\end{tabular}

\section{DISCUSSION}

While a number of studies have explored statinassociated adverse effects, the lack of standard definitions of statin intolerance and associated symptoms [3], as well as nonuniformity of the outcomes measured, make it difficult to identify the patients having difficulty with their statin treatment and, more importantly, those at risk for discontinuing their treatment because of the difficulties [11]. Our literature search revealed a notable lack of patient-centered research in the field. There are currently no validated patientreported outcome measures to assess the symptoms or impacts of statin intolerance. With the introduction of new lipid-lowering therapies for the treatment of dyslipidemias, it is increasingly important to identify patients having difficulties with their statin treatment to ensure timely and clinically beneficial treatment modifications [12]. Our goal is to develop a patient survey to describe the patient experience with statin intolerance to inform clinical practice and assist with identification of patients who may need extra attention to help keep them on a treatment regimen.

This qualitative study identified key symptoms and impacts associated with statin intolerance that were highly relevant to the patient experience. Symptoms of muscle-related pain or discomfort were the most commonly reported statin-related symptoms reported by patients, appearing in $77 \%$ of interviews and comprising $28 \%$ of symptom expressions. Impacts on physical functioning were the most commonly reported statin-related impacts reported by patients, representing 36\% of impact expressions. These observations were consistent with information obtained from the literature review and from clinician interviews. However, the results also showed that the symptom and impact experience from the patient perspective is broader than currently reflected in the literature and assumed by clinicians. While not all of the concepts expressed will be reflected in the survey, those with relevance to larger numbers of the patients interviewed will be included.

Ultimately, results from the patient survey will support clinician awareness for effective decision-making. Evaluation of symptom severity and impact severity will assist with describing the patients who experience a greater symptom and impact burden and have a higher risk of giving up and discontinuing their treatment. While statin side effect histories provide a clinically descriptive picture of the patient's journey through the difficulties with statin treatment, these histories can also provide key information regarding tendencies toward continuing or discontinuing statin use. Other key elements in the conceptual framework are expected to provide insight into which patient characteristics might indicate a possible association with discontinuing statin treatment, and help clinicians identify possible ways to assist those patients in staying on treatment.

Qualitative studies have inherent limitations, including the possibility of selection bias 
in the recruitment of sites and patients. The inclusion criteria were selected to intentionally focus on patients who had a recent experience with statins and who returned to their doctor reporting difficulties as a result of the statin. While the patients selected across sites may not be representative of all patients having difficulties with their statins, the group interviewed were from a variety of countries and sites, and the saturation evaluation provides good evidence that sufficient representation of the key concepts from this population came forward in the interview process and was considered.

\section{CONCLUSIONS}

Qualitative interviews prompted patients to address a wider range of symptoms and impacts than are usually discussed in clinic visits. A better understanding of the symptoms and impacts that patients experience with statin use may assist clinicians with managing individual patients in a way that provides options that encourage adherence to treatment. The next steps of this research will be to confirm the clarity and understandability of the items in the survey using cognitive interviews, and then to use the survey to obtain a quantitative dataset that can be used to address the outstanding questions of the study.

\section{ACKNOWLEDGEMENTS}

The authors thank the patients who participated in this study. This study was the result of a collaboration between the European Atherosclerosis Society (EAS), the study sponsor (Amgen Inc.), and Health Research Associates, Inc. The EAS Steering Committee provided overall direction and clinical expertise in the design of the study, and contributed to the interpretation of the study results and development of the patient survey. The Steering Committee also recruited EAS clinicians in the participating countries to take part in the expert interviews and provide patients from their clinic settings to take part in the qualitative interviews. We would like to acknowledge the following clinicians for their assistance with this project: Jan Pitha (Czech Republic), Vladimir Blaha (Czech Republic), Maurizio Averna (Italy), Alberico Catapano (Italy), Cecilie Wium (Norway), Daniel Pella (Slovakia), Branislav Vohnout (Slovakia), Jose Zamorano (Spain), Luis Masana (Spain), Stefano Romjeo (Sweden), Jan Alvang (Sweden), and Jaimini Cegla (United Kingdom).

Funding. This study and the cost of publication (Rapid service and Open Access fees) were sponsored by Amgen Inc.

Medical Writing Assistance. Editorial assistance in the preparation of this article was provided by Dr. Julia R. Gage of Gage Medical Writing, LLC. Support for this assistance was funded by Amgen Inc.

Authorship. All named authors meet the International Committee of Medical Journal Editors (ICMJE) criteria for authorship for this article, take responsibility for the integrity of the work as a whole, and have given their approval for this version to be published.

Author contributions. Michal Vrablik contributed to data acquisition, and analysis and interpretation of the data. Alberico Catapano contributed to study design, data acquisition, and analysis and interpretation of the data. Olov Wiklund contributed to data acquisition, and analysis and interpretation of the data. Yi Qian contributed to study design, and analysis and interpretation of the data. Pratik Rane contributed to analysis and interpretation of the data. Alyson Grove contributed to data acquisition and selection of content for the survey. Mona L. Martin contributed to study design, overall study management, data acquisition, and analysis and interpretation of the data. All authors contributed to the writing of the manuscript and all authors approved the draft for submission. All authors had full access to all of the data in this study and take complete responsibility for the integrity of the data and accuracy of the data analysis. 
Disclosures. Michal Vrablik has received research support and consulting fees from Amgen, Pfizer, Sanofi, and MSD; and nonfinancial support from Amgen and Sanofi. Alberico Catapano has received grant support from Pfizer, Sanofi, Regeneron, Merck, and Mediolanum; consulting fees from Aegerion, Amgen, AstraZeneca, Genzyme, Bayer, SigmaTau, Menarini, Kowa, Eli Lilly, Recordati, Pfizer, Sanofi, Mediolanum, and Merck; and nonfinancial support from SigmaTau, Menarini, Kowa, Recordati, and Eli Lilly. Olov Wiklund has received honoraria for lecturing from Amgen and Sanofi. Yi Qian is an employee and shareholder of Amgen Inc. Pratik Rane is an employee and shareholder of Amgen Inc. Alyson Grove has nothing to disclose. Mona Martin was an employee of Health Research Associates, Inc. at the time of this study, which received funding from Amgen to conduct this study; is now currently affiliated with Evidera, 1417 4th Avenue, Suite 510, Seattle, Washington, 98101, USA

Compliance with Ethics Guidelines. Ethics committee approval was obtained for each study site (Supplemental List S1), and all participants provided written informed consent prior to initiation of any study activity.

Data Availability. Datasets from this study are proprietary and are not publicly available.

Open Access. This article is distributed under the terms of the Creative Commons Attribution-NonCommercial 4.0 International License (http://creativecommons.org/licenses/ by-nc/4.0/), which permits any noncommercial use, distribution, and reproduction in any medium, provided you give appropriate credit to the original author(s) and the source, provide a link to the Creative Commons license, and indicate if changes were made.

\section{REFERENCES}

1. Ito MK. Dyslipidemia: management using optimal lipid-lowering therapy. Ann Pharmacother. 2012;46:1368-81.
2. Health-at-a-glance-2013. Available from: https:// www.oecd.org/els/health-systems/Health-at-a-Glan ce-2013.pdf. Accessed 23 Aug 2019.

3. Algharably EA, Filler I, Rosenfeld S, Grabowski K, Kreutz R. Statin intolerance-a question of definition. Expert Opin Drug Saf. 2017;16:55-63.

4. Stroes ES, Thompson PD, Corsini A, et al. Statinassociated muscle symptoms: impact on statin therapy-European Atherosclerosis Society Consensus panel statement on assessment, aetiology and management. Eur Heart J. 2015;36:1012-22.

5. Muntean DM, Thompson PD, Catapano AL, et al. Statin-associated myopathy and the quest for biomarkers: can we effectively predict statin-associated muscle symptoms? Drug Discov Today. 2017;22:85-96.

6. Ganga HV, Slim HB, Thompson PD. A systematic review of statin-induced muscle problems in clinical trials. Am Heart J. 2014;168:6-15.

7. Vonbank A, Drexel H, Agewall S, et al. Reasons for disparity in statin adherence rates between clinical trials and real-world observations: a review. Eur Heart J Cardiovasc Pharmacother. 2018;4:230-6.

8. Tuccori M, Lapi F, Testi A, et al. Statin-associated psychiatric adverse events: a case/non-case evaluation of an Italian database of spontaneous adverse drug reaction reporting. Drug Saf. 2008;31:1115-23.

9. Mampuya WM, Frid D, Rocco M, et al. Treatment strategies in patients with statin intolerance: the Cleveland Clinic experience. Am Heart J. 2013;166:597-603.

10. Kutner JS, Blatchford PJ, Taylor DH Jr, et al. Safety and benefit of discontinuing statin therapy in the setting of advanced, life-limiting illness: a randomized clinical trial. JAMA Intern Med. 2015;175:691-700.

11. Alonso R, Cuevas A, Cafferata A. Diagnosis and management of statin intolerance. J Atheroscler Thromb. 2019;26:207-15.

12. Koskinas $\mathrm{K}$, Wilhelm $\mathrm{M}$, Windecker S. Current treatment of dyslipidaemia: PCSK9 inhibitors and statin intolerance. Swiss Med Wkly. 2016;146: w14333. 